\title{
Deconfinement and chiral symmetry restoration in QCD with fundamental and adjoint fermions
}

\author{
Frithjof Karsch \\ Fakultät für Physik, Universität_Bielefeld,_D-33615 Bielefeld, Germany \\ E-mail: kkarsch@physik. uni-bielefeld.dei
}

ABstract: We discuss the critical behaviour of strongly interacting matter close to the QCD phase transition. Emphasis is put on a presentation of results from lattice calculations that illustrate deconfining as well as chiral symmetry restoring features of the phase transition. We show that both transitions coincide in QCD while they fall apart in an $\mathrm{SU}(3)$ gauge theory coupled to adjoint fermions.

\section{Introduction}

The interest in analyzing the properties of QCD at non-zero temperature is twofold. On the one hand it is the goal to reach a quantitative description of the behaviour of matter at high temperature and density. This does provide important input for a quantitative description of experimental signatures for the occurrence of a phase transition in heavy ion collisions and should also help to understand better the phase transitions that occurred during the early times of the evolution of the universe. Eventually it also may allow to answer the question whether a quark-gluon plasma can exist in the interior of dense neutron stars. For this reason one would like to reach a quantitative understanding of the QCD equation of state, determine critical parameters such as the critical temperature and the critical energy density and predict the modification of basic hadron properties (masses, decay widths) with temperature and density. On the other hand the analysis of a complicated quantum field theory at non-zero temperature can also help to improve our understanding of its non-perturbative properties at zero temperature. The introduction of an external control parameter (temperature) allows to observe the response of different observables to this and may provide a better understanding of their interdependence [i1] ular, one would like to clarify the role of confine- ment and chiral symmetry breaking for the QCD phase transition.

In which respect is the QCD phase transition deconfining and/or chiral symmetry restoring? In the next section we will address this question and will present some basic results on the QCD equation of state and critical parameters at the transition point obtained from lattice QCD. In section 3 we will discuss deconfinement and chiral symmetry restoration in an $\mathrm{SU}(3)$ gauge theory with adjoint fermions. In section 5 we give our conclusions. Throughout this write-up we will as far as possible try to avoid going into details of the actual lattice calculations. Basics concepts of the lattice formulation of QCD relevant for finite temperature calculations and in particular for the discussion of deconfinement and chiral symmetry restoration can be found for instance in Refs. 2 and 3.

\section{The QCD phase transition}

Two properties of QCD explain the basic features of the observed spectrum of hadrons - confinement and chiral symmetry breaking. While the former explains why we observe only colourless states in the spectrum the latter describes the presence of light Goldstone particles, the pions. The confining property of QCD manifests itself in the large distance behaviour of the heavy quark potential. At zero temperature the poten- 


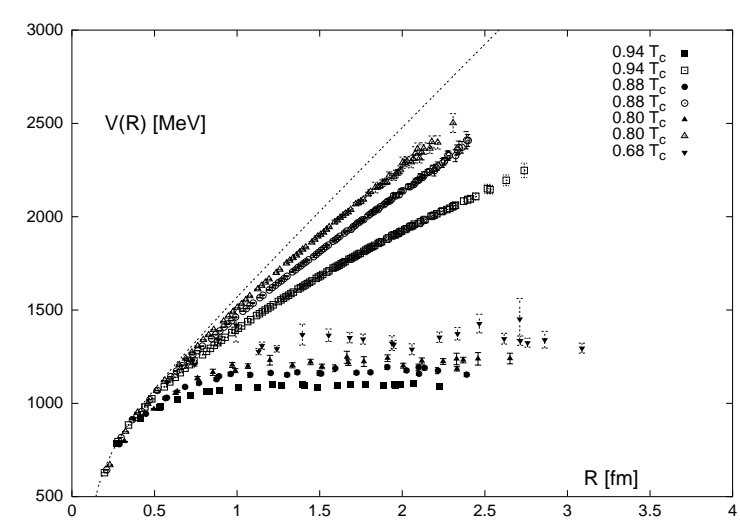

Figure 1: The heavy quark potential in 2-flavour QCD with quarks of mass $m / T=0.15$ extracted from calculations on a $16^{3} \times 4$ lattice and in quenched QCD on $32^{3} \times 4$ lattices. The dashed line shows results for the potential at $T \simeq 0$.

tial rises linearly at large distances, $V_{\bar{q} q}(r) \sim \sigma r$, where $\sigma \simeq(420 \mathrm{MeV})^{2}$ denotes the string tension, and forces the quarks and gluons to be confined in a hadronic bag. Chiral symmetry breaking leads to a non-vanishing quark anti-quark condensate, $\langle\bar{q} q\rangle \simeq(250 \mathrm{MeV})^{3}$, in the vacuum. Inside the hadron bag, however, the condensate vanishes. At high temperatures the individual hadronic bags are expected to merge to a single large bag, in which quarks and gluons can move freely. This bag picture provides some intuition for the occurrence of deconfinement and chiral symmetry restoration. A priory it is, however, not evident that confinement and the broken chiral symmetry have to get lost at the same temperature. It has been speculated that two distinct phase transitions leading to deconfinement at $T_{d}$ and chiral symmetry restoration at $T_{\chi}$ could occur in QCD [i $\left[\begin{array}{l}-1 \\ 4\end{array}\right]$. General arguments about the scales involved suggest that $T_{d} \leq T_{\chi}$.

In fact, the discussion of confinement and deconfinement in terms of the heavy quark potential as presented above makes sense only in the limit of heavy quarks. For light quarks the spontaneous creation of quark anti-quark pairs from the vacuum leads to a breaking of the "string" between static quark sources, i.e. the potential tends to a constant value for $r \rightarrow \infty$. In particular at large temperature the distinction between confinement and deconfinement thus seems to be- come a qualitative one. This is evident from a comparison of heavy quark potentials calculated in QCD with light quarks as well as in the quenched limit at temperatures close but below the phase transition $[5]$. The potentials shown in Figure ${ }_{1}^{1}$ have been obtained from a calculation of expectation values of the Polyakov loop correlation function

$$
\exp \left(-\frac{V_{\bar{q} q}(r, T)}{T}\right)=\left\langle\operatorname{Tr} L_{\vec{x}} \operatorname{Tr} L_{\vec{y}}^{\dagger}\right\rangle,
$$

where $L_{\vec{x}}$ and $L_{\vec{y}}^{\dagger}$ represent a static quark and anti-quark pair ${ }^{1}$ located at the spatial points $\vec{x}$ and $\vec{y}$, respectively, $r=|\vec{x}-\vec{y}|$.

At large distances the Polyakov loop correlation function approaches $|\langle L\rangle|^{2}$, where $L$ is given by $L=N_{\sigma}^{-3} \sum_{\vec{x}} \operatorname{Tr} L_{\vec{x}}$. The Polyakov loop expectation value $\langle L\rangle$ thus reflects the behaviour of the heavy quark potential at large distances although it is not an order parameter in the strict sense. A sudden rise in $\langle L\rangle$ indicates that the heavy quark potential flattens already at even shorter distances; the magnitude of $\langle L\rangle$ thus still signals the transition from a predominantly confining to a deconfining thermal medium. This transition also manifests itself in the occurrence of a peak in the Polyakov loop susceptibility,

$$
\chi_{L}=\left\langle L^{2}\right\rangle-\langle L\rangle^{2}
$$

The temperature dependence of chiral properties of QCD on the other hand becomes visible directly through the temperature dependence of the chiral condensate, $\langle\bar{\psi} \psi\rangle$ as well as its derivative with respect to the quark mass, the chiral susceptibility, $\chi_{m}$,

$$
\chi_{m}=\frac{\partial}{\partial m_{q}}\langle\bar{\psi} \psi\rangle .
$$

Results from a calculation of these observables for QCD with two light quarks [i] $[\bar{i}]$ are shown in Figure 2 , as a function of the lattice bare coupling $\beta=6 / g^{2}$. Evidently the peak in both susceptibilities is located at the same value of the (pseudo)-critical couplings, $\beta_{c}\left(m_{q}\right)$. This indicates that the transition to a deconfined, chirally symmetric phase occurs at the same temperature. These results provide the basic evidence

\footnotetext{
${ }^{1}$ Further details on the definition of this observable is given for instance in Refs. 2 and 3.
} 

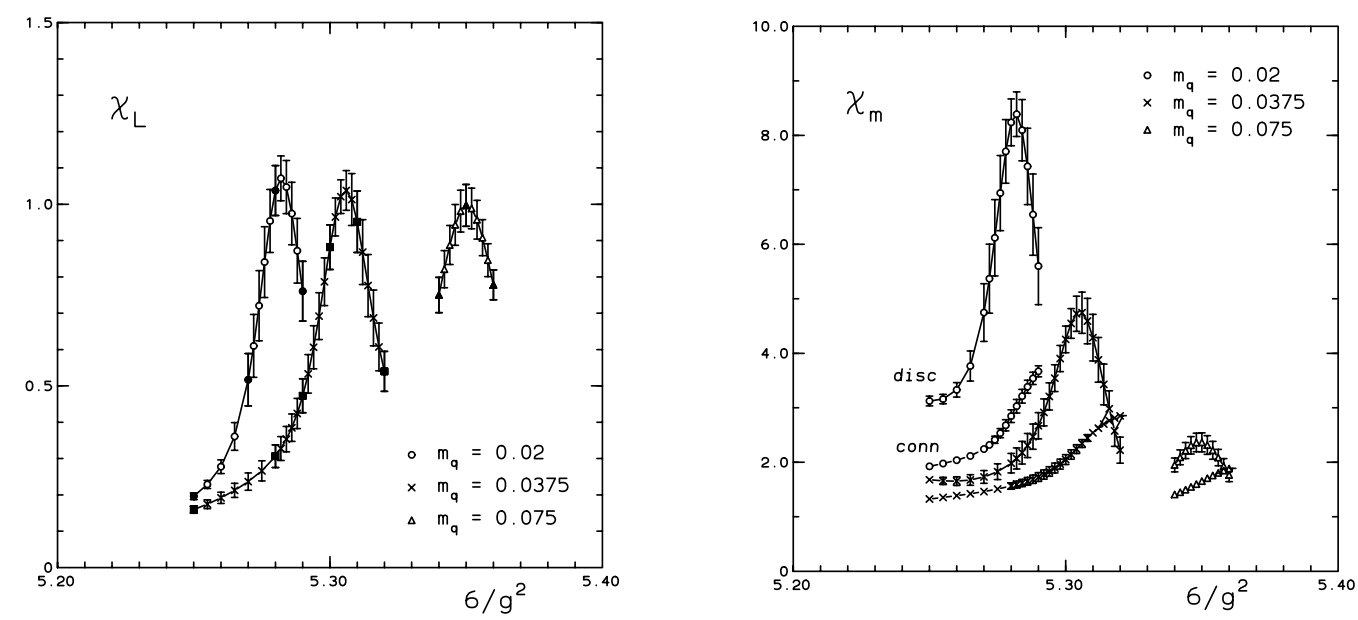

Figure 2: Polyakov loop and chiral susceptibilities versus $\beta=6 / g^{2}$ in 2-flavour QCD for several values of the quark mass calculated on lattices of size $8^{3} \times 4$.

for the existence of a single phase transition in QCD.

In fact, the finite peak heights of the susceptibilities shown in Figure but smooth variation of $\langle\bar{L}\rangle$ and $\langle\bar{\psi} \psi\rangle$ itself do not yet correspond to a true phase transition. This would be signaled by diverging susceptibilities and is expected to occur only in the infinite volume and zero quark mass limit ${ }^{2}$,

$$
\chi_{m} \sim m_{q}^{1 / \delta-1},
$$

where $\delta$ is a critical exponent. Although not yet confirmed through numerical simulations it is expected that the exponent $\delta$ will in the case of 2-flavour QCD be equal to that of 3dimensional, $O(4)$ symmetric spin models, $\delta \simeq$ 4.82 [1] 0 ]. We note that the chiral susceptibility indeed grows rapidly with decreasing quark mass while the Polyakov loop susceptibility shows little variation with $m_{q}$ in the quark mass regime covered in Figure $\overline{2}$ In fact, for these quark masses $\chi_{L}$ also does not change significantly with increasing spatial volume while it would rise rapidly $(\sim V)$ in the heavy quark mass regime where a first order deconfinement transition occurs.

\footnotetext{
${ }^{2}$ This is correct only when the phase transition is second order. In the case of a first order transition the susceptibilities would diverge already for masses below a certain non-zero quark mass. This will happen, for instance, in the case of QCD with three light flavours [7,' 84.
}

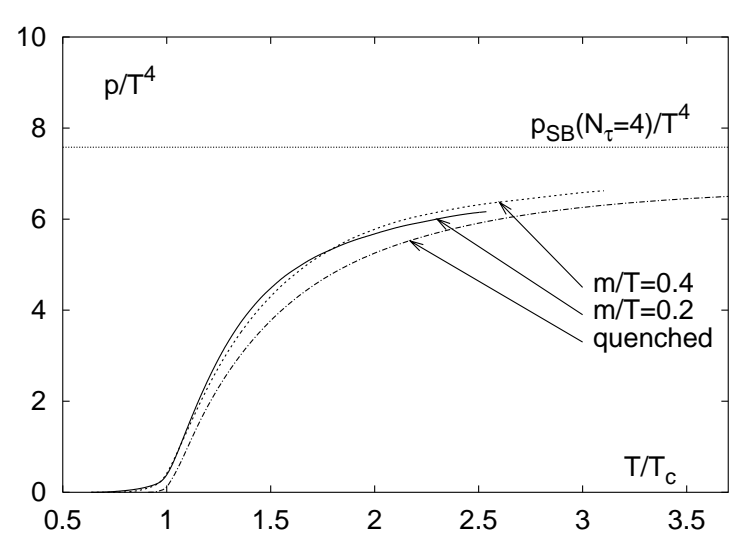

Figure 3: The pressure in units of $T^{4}$ versus $T / T_{c}$ for a pure $\mathrm{SU}(3)$ gauge theory (quenched) and four flavour QCD with two different values of the quark mass $(m / T=0.2,0.4)$. The numerical results for four flavour QCD have been rescaled by the corresponding ratio of ideal gas values, $p_{\mathrm{SB}}\left(n_{f}=\right.$ $4) / p_{\mathrm{SB}}\left(n_{f}=0\right)$.

We conclude that observables directly related to chiral symmetry restoration show critical behaviour in the zero quark mass limit, while observables related to deconfinement do not seem to become singular in this limit. In this respect it may be justified to call the QCD phase transition a chiral phase transition. However, this is only one feature of the transition. The deconfin- 
ing aspect of the transition is particularly evident when looking at the behaviour of bulk thermodynamic observables like the energy density or the pressure. Asymptotically, for $T \rightarrow \infty$, these quantities approach the value of an ideal gas and thus directly reflect the number of light degrees of freedom in the system. For QCD with $n_{f}$ light (massless) quarks and anti-quarks as well as $N_{c}^{2}-1$ gluons $\left(N_{c}=3\right)$ the pressure and energy density approach the Stefan-Boltzmann value,

$$
\frac{p_{\mathrm{SB}}}{T^{4}}=\frac{1}{3} \frac{\epsilon_{\mathrm{SB}}}{T^{4}}=\frac{\pi^{2}}{45}\left(N_{c}^{2}-1+\frac{7}{4} N_{c} n_{f}\right),
$$

which directly counts the relevant number of light degrees of freedom. Above $T_{c}$ these degrees of freedom get liberated which is reflected in a rapid rise of the energy density and the pressure. In Figure $\overline{3}$, we show a comparison of the temperature dependence of the pressure calculated in the $\mathrm{SU}(3)$ gauge theory [1 $\left.1 \mathrm{i}_{1}^{\prime}\right]\left(n_{f}=0\right)$ and four flavour QCD [12in $\left(n_{f}=4\right)$. In both cases the high temperature limits differ by more than a factor of three. Nonetheless, when normalized to the corresponding Stefan-Boltzmann values the pressure $p / p_{\mathrm{SB}}$ shows a similar temperature dependence. Similar conclusions can also be drawn from an analysis of the energy density. Bulk thermodynamic observables thus show that the light partonic degrees of freedom indeed get liberated at $T_{c}$. Their sudden increase reflects the onset of deconfinement.

\section{SU(3) gauge theory with adjoint fermions}

To some extent the existence of a single phase transition in QCD may not be too surprising. After all the QCD Lagrangian has only one global symmetry - chiral symmetry, which is spontaneously broken in the limit of vanishing quark mass. Only in the pure gauge limit, i.e. the limit of infinitely heavy quarks there exists another exact symmetry, the $\mathrm{Z}(3)$ center symmetry, which is related to confinement in the literal sense that $V_{\bar{q} q}(r, T)$ approaches infinity for $r \rightarrow \infty$. The $\mathrm{Z}(3)$ symmetry does get spontaneously broken at the critical temperature of the $\mathrm{SU}(3)$ gauge theory. For all finite values of the quark mass it is, however, explicitly broken. The

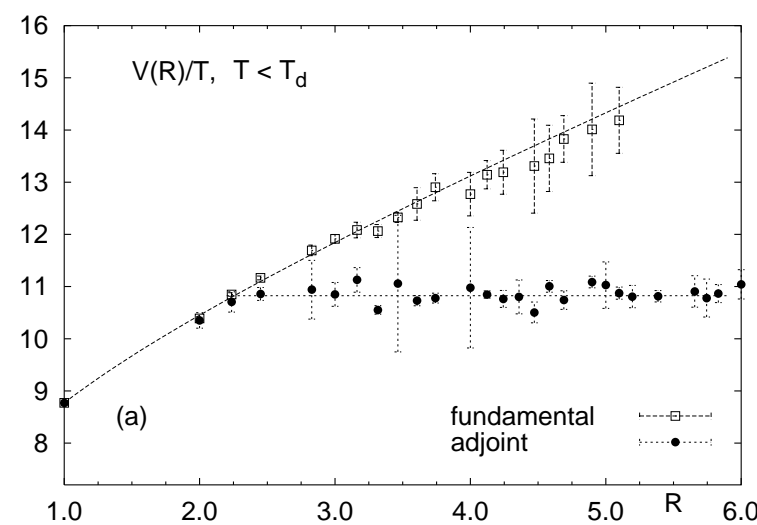

Figure 4: The potential between fundamental and adjoint static quark sources in the low temperature phase of aQCD. While the potential is confining for fundamental charges it is not for adjoint charges.

importance of the realization of an exact center symmetry in the Lagrangian for the existence of a true phase transition related to deconfinement can be analyzed in a QCD-related model like the $\mathrm{SU}(3)$ gauge theory with fermions in the adjoint rather than in the fundamental represen-

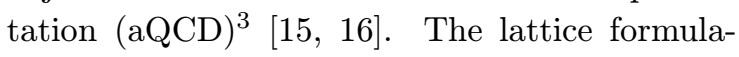
tion of aQCD is obtained from that of ordinary QCD by replacing the three-dimensional representation of the gauge link matrices, $U^{(3)}$, in the fermionic part of the action by the corresponding eight-dimensional representation, $U^{(8)}$,

$$
\begin{aligned}
S_{\mathrm{aQCD}} & =S_{G}+S_{F}\left(U^{(8)}\right) \\
\text { with } \quad U_{a, b}^{(8)} & =\frac{1}{2} \operatorname{Tr} U^{(3)} \lambda_{a} U^{(3)} \lambda_{b} .
\end{aligned}
$$

At zero temperature this theory has a broken chiral symmetry and an exact $\mathrm{Z}(3)$ center symmetry, i.e. for all values of the adjoint fermion mass it is strictly confining for fundamental charges which are used to probe the heavy quark potential. What is less obvious though is that the $\mathrm{Z}(3)$ symmetry plays any role for the deconfinement of the dynamical degrees of freedom participating in the thermodynamics, in particular for

\footnotetext{
${ }^{3}$ We note that the model we are discussing here is closely related to supersymmetric gauge theories. For a recent attempt to simulate supersymmetric Yang Mills theories on the lattice see [13]. The possibility of the breaking of the $Z(N)$ center symmetry in finite temperature supersymmetric models has recently also been discussed in Ref. [14]
} 

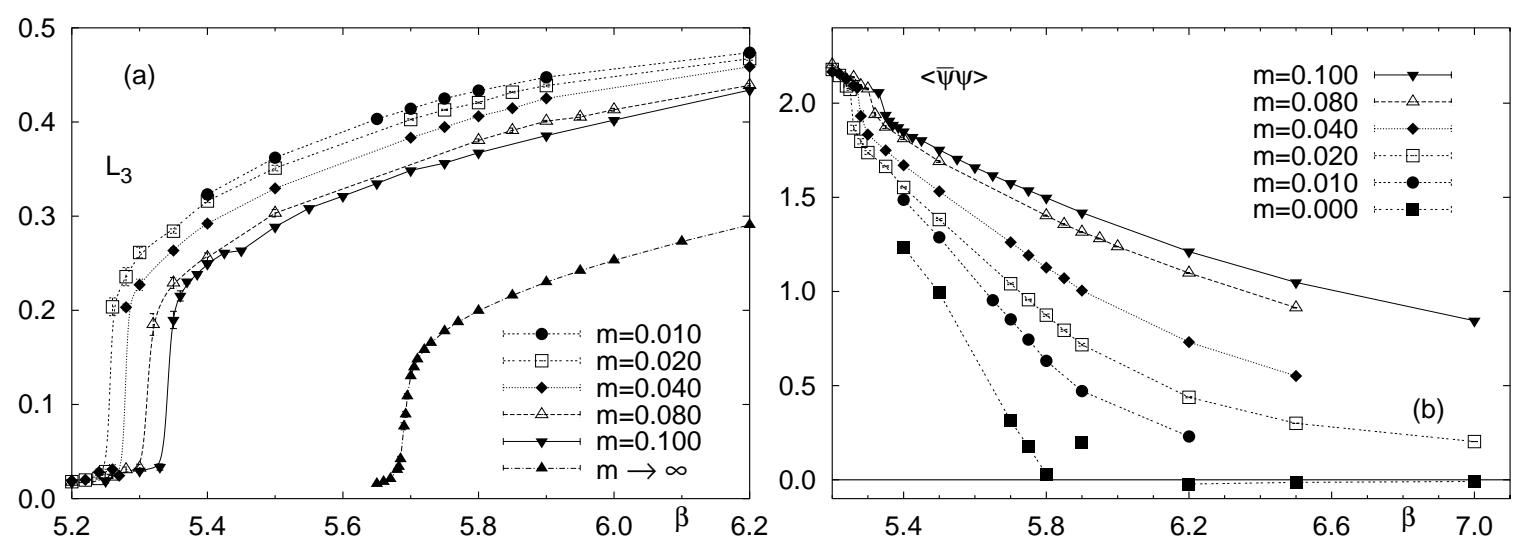

Figure 5: Polyakov loop expectation value, $L_{3}$, in the fundamental representation obtained from simulations on $8^{3} \times 4$ lattices with adjoint fermions of various masses (left) and the fermion condensate (right) obtained with the same simulation parameters.

the adjoint quarks which are blind to the center symmetry. In fact, the potential between static charges in the adjoint representation is not confining; at large distances string breaking occurs as is evident from Figure 'í.

In Figure, $5, \overline{5}$ we show the Polyakov loop expectation values for fundamental and adjoint charges as well as the fermion condensate. It is evident that there are two distinct phase transitions, i.e. deconfinement occurs before chiral symmetry restoration $\left(T_{\chi} \simeq 8 T_{d}\right)$ [1] ${ }_{1}^{\prime}$.

Contrary to QCD the $\mathrm{SU}(3)$ gauge theory with adjoint quarks has an intermediate phase where confinement is lost but chiral symmetry remains broken. It is apparent from Figure in this phase the chiral condensate is much more sensitive to changes of the fermion mass than in the confined phase. In fact, for $T_{d}<T<T_{\chi}$ the leading fermion mass dependence of the chiral condensate is

$$
\langle\bar{\psi} \psi\rangle=a_{0}+a_{1} m^{1 / 2}+\mathcal{O}(m)
$$

whereas below $T_{d}$ the leading order correction seems to start only at $\mathcal{O}(m)$. This is even more evident from the chiral susceptibility of aQCD shown in Figure $\overline{6}$. In the intermediate phase $\chi_{m}$ shows a strongly quark mass dependent plateau which diverges like $m^{-1 / 2}$. The occurrence of such a divergence in the chiral susceptibility below but close to $T_{\chi}$ has been expected. It was shown to exist in $3 d$ sigma models where it arises from fluctuations of Goldstone modes in the unbroken directions [1] $\left.\overline{7}_{1}\right]$. We thus find that universality indeed links the chiral properties of $(3+1)$ dimensional aQCD below $T_{\chi}$ to those of $3 d$ sigma models. To establish this relation also directly at $T_{\chi}$ seems to be more difficult. Here a more rapid divergence, $\chi_{m} \sim m^{1 / \delta-1} \sim m^{-0.8}$, is expected. Although the simulation results shown in Figure $\overline{6}_{1}^{1}$ indicate such a more rapid divergence at $T_{\chi}$, the corresponding peak in $\chi_{m}$ shows up only for rather small quark masses and a reliable determination of critical indices is not yet possible.

A similar behaviour is expected to occur in QCD. So far the studies of chiral properties at the QCD phase transition, however, did not yield the critical indices expected on the basis of universality arguments $\left[\overline{6}_{\mathrm{b}}^{1}, \overline{9} \overline{1}\right]$. From the study of chiral symmetry breaking in aQCD one might conclude that the occurrence of additional subleading singularities as well as the strong influence of confinement on the quark mass dependence of chiral observables do make a more complex analysis of chiral observables in QCD necessary. One probably has to take into account also subleading dependences on the quark mass.

\section{Conclusions}

Strongly interacting matter does undergo a single phase transition at finite temperature, which 


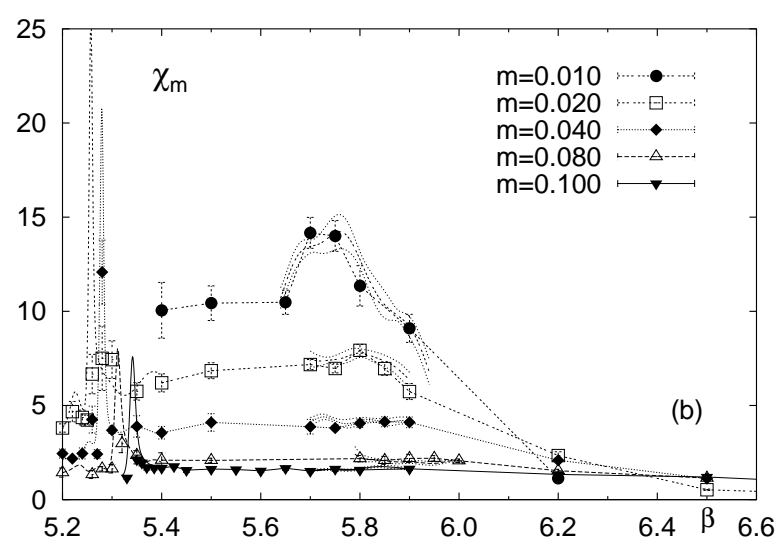

Figure 6: Polyakov loop susceptibility of aQCD versus $\beta$ for several values of the fermion mass.

is deconfining and chiral symmetry restoring. We have discussed some aspects of this transition which show its chiral symmetry restoring as well as deconfining properties.

The chiral order parameter and its susceptibility show a strong quark mass dependence which signals the occurrence of a phase transition in the zero quark mass limit. However, the details of this quark mass dependence are in the case of two-flavour QCD so far not in agreement with the expected universal behaviour of the 3 -dimensional $O(4)$ symmetric sigma-models. Nonetheless the transition seems to be continuous, at least in the quark mass regime so far accessible to numerical calculations $\left(m_{q} / T \gtrsim 0.04\right)$ there are no indications for a first order transition.

The analysis of a $\mathrm{SU}(3)$ gauge theory with adjoint fermions shows that two distinct phase transitons, related to deconfinement and chiral symmetry restoration, can occur in quantum field theories at finite temperature. The exact realization of the $Z(3)$ center symmetry seems to be essential for this. In the case discussed here a deconfining transiton occurs at a lower temperature $T_{d}$ than the chiral transition temperature $T_{\chi}$. Chiral observables exhibit universal scaling behaviour close to $T_{\chi}$ as expected from the analysis of $3 d$ sigma models. Below the deconfinement transition temperature, $T_{d}$, confinement dominates and these singularities are no longer visible. This indicates that even close to $T_{\chi}$ the chiral sector of QCD may be influenced strongly by confinement as well as by contributions from subleading chiral singularities.

\section{Acknowledgments}

We gratefully acknowledge partial support through the TMR-network "Finite Temperature Phase Transitions in Particle Physics", EU contract no. ERBFMRX-CT97-0122.

\section{References}

[1] F. Wilczek, Back to basics at ultrahigh temperatures, Physics Today, April 1998, p.11

[2] F. Karsch, Simulating the Quark-Gluon Plasma on the Lattice, Advanced Series on Directions in High Energy Physics - Vol.6 (1990) 61, "Quark Gluon Plasma" (Edt. R.C. Hwa), Singapore 1990, World Scientific.

[3] K. Kanaya, An introduction to finite temperature Quantumchromodynamics on the lattice, Prog. Theor. Phys. Suppl. 131 (1998) 73.

[4] E. Shuryak, Phys. Lett. B107 (1981) 103.

[5] C. DeTar, O. Kaczmarek, F. Karsch and E. Laermann, Phys. Rev. D59 (1999) 031501.

[6] F. Karsch and E. Laermann, Phys. Rev. D50 (1994) 6954.

[7] A.Aoki et al. (JLQCD Collaboration), Nucl. Phys. B (Procl. Suppl.) 73 (1999) 459.

[8] A. Peikert, F. Karsch, E. Laermann and B. Sturm, Nucl. Phys. B (Procl. Suppl.) 73 (1999) 468.

[9] S. Aoki et al., Phys. Rev. D57 (1998) 3910.

[10] , K. Kanaya and S. Kaya, Phys. Rev. D51 (1995) 2404.

[11] G. Boyd et al., Phys. Rev. Lett. 75 (1995) 4169 and Nucl. Phys. B469 (1996) 419.

[12] J. Engels et al., Phys. Lett. B396 (1997) 210.

[13] R. Kirchner, S. Luckmann, I. Montvay, K. Spanderen and J. Westphalen, Phys. Lett. B446 (1999) 209.

[14] E. Witten, Adv. Theor. Math. Phys. 2 (1998) 505.

[15] J. Kogut, J. Polonyi, D.K. Sinclair and H. W. Wyld, Phys. Rev. Lett. 54 (1985) 1980.

[16] F. Karsch and M. Lütgemeier, Nucl. Phys. B550 (1999) 449.

[17] D.J. Wallace and R.K.P. Zia, Phys. Rev. B12 (1975) 5340. 